Revista Colombiana de Obstetricia y Ginecología Vol. 62 No. 4 • Octubre-Diciembre 2011 •(326-330)

Reporte de caso

\title{
ESTRUMOSIS PERITONEAL: REPORTE DE CASO Y REVISIÓN DE LA LITERATURA
}

\author{
Peritoneal strumosis: a case report and literature \\ review \\ Javier Baena-Del Valle, M.D. *, Joan Gutiérrez-Sanmartín, M.D. *, \\ Katherine Redondo-De Oro, M.D. *, César Redondo-Bermúdez, M.D. ** \\ Recibido: diciembre 16/10 - Aceptado: noviembre 29/11
}

\section{RESUMEN}

Objetivo: se presenta un caso de estrumosis peritoneal para hacer una revisión de la literatura publicada acerca de las características histológicas y clínicas de estos tumores.

Presentación del caso: paciente de 48 años de edad con historia de ooforectomía izquierda hace 34 años por tumor ovárico benigno, consulta al Hospital Universitario del Caribe, centro de referencia del departamento de Bolívar, por presentar dolor en hipocondrio izquierdo.

Examen físico: masa abdominal confirmada mediante ultrasonido y tomografía computarizada abdominal. Se encontró masa retroperitoneal de 15,5 x 10,5 x $7 \mathrm{~cm}$, en la laparotomía exploratoria, la cual se resecó. El análisis microscópico reveló tejido tiroideo constituido por folículos con estructura típica, lo cual indicó una estrumosis peritoneal.

Materiales y métodos: se realizó una revisión bibliográfica en cinco bases de datos (Medline, Ebsco, PubMed, Science Direct y Cochrane Reviews), utilizando como encabezados de búsqueda [Título/Resumen]: peritoneal struma, peritoneal strumosis, struma ovarii. Se incluyeron 15 citas bibliográficas.

Médico, Residente de Patología. Facultad de Medicina, Universidad de Cartagena. Cartagena (Colombia).

** Médico Patólogo, Docente, Sección de Patología, Departamento de Diagnóstico. Facultad de Medicina, Universidad de Cartagena. Cartagena (Colombia). Correo electrónico: crbpatologo@yahoo.com.mx
Conclusiones: la estrumosis peritoneal es una lesión poco frecuente, asociada al teratoma quístico maduro del ovario, cuyo diagnóstico preoperatorio es difícil. Este es un nuevo caso publicado en la literatura mundial.

Palabras clave: estrumosis peritoneal, estruma ovárico, adenocarcinoma folicular, ovario, tiroides.

\section{SUMMARY}

Objective: a case of peritoneal strumosis is presented for making a review of the literature published about these histological tumors and their clinical characteristics.

Case presentation: a 48 -year-old patient, having a history of left side oophorectomy 34 beforehand due to a benign ovarian tumor, consulted at the Caribe Teaching Hospital (a reference centre for the Bolívar department) as she had pain in the left hypochondrium.

Physical exam: abdominal mass confirmed by ultrasound and abdominal computerized tomography. A $15.5 \times 10.5 \times 7 \mathrm{~cm}$ retroperitoneal mass was found during exploratory laparotomy which was removed. Microscope analysis revealed thyroid tissue consisting of follicles having typical structure, constituting a peritoneal strumosis.

Materials and methods: a literature review was made of five databases (Medline, Emsco, PubMed, Science Direct and Cochrane Reviews), using search keywords [Title/Summary]: "peritoneal struma", 
"peritoneal strumosis" ad "struma ovarii"; 15 bibliographical citations were included.

Conclusions: peritoneal strumosis is an infrequently occurring lesion which is associated with mature cystic teratoma of the ovary, whose pre-operation diagnosis is difficult. This is a new case published in the worldwide literature.

Key words: peritoneal strumosis, struma ovarii, follicular adenocarcinoma, ovary, thyroid.

\section{INTRODUCCIÓN}

La estrumosis peritoneal es definida como la presencia de implantes constituidos por tejido tiroideo maduro en el peritoneo. ${ }^{1,2}$ No se conoce con exactitud la frecuencia de esta patología, pero si se ha establecido su relación con tumores ováricos, especialmente teratomas quísticos maduros, los cuales representan el 40 - 50\% de las neoplasias ováricas; el 0,3 - 1\% de estos son estrumas ováricos, el teratoma monodérmico más común del ovario, ${ }^{3}$ puede ser clasificado como benigno o maligno. ${ }^{3}$ En raras ocasiones, el tejido tiroideo benigno puede diseminarse a la cavidad peritoneal, dando origen a una estrumosis peritoneal.

Consideramos que es un tema de interés para los lectores debido a que llama la atención sobre la variedad de diagnósticos diferenciales que se deben considerar durante la evaluación del paciente con una masa abdominal y la relevancia de una buena historia clínica y de las técnicas imagenológicas en el abordaje de estos. Presentamos un caso de estrumosis peritoneal en el Hospital Universitario del Caribe en Cartagena de Indias (Colombia), con el objetivo de hacer una revisión de la literatura publicada acerca de las características histológicas y clínicas de estos tumores.

\section{REPORTE DE CASO}

Paciente de 48 años de edad, soltera, quien consulta por presentar dolor abdominal en hipocondrio izquierdo irradiado a región lumbar, de un mes de evolución, antecedentes de hipertensión arterial controlada con captopril e hidroclorotiazida, ooforectomía izquierda a la edad de 14 años por tumor ovárico benigno e histerectomía abdominal total a los 30 años por miomatosis uterina. Es remitida al Hospital Universitario del Caribe, centro de referencia en la ciudad de Cartagena (Colombia), que atiende a la población del régimen subsidiado y estratos socioeconómicos medios y bajos. El examen físico reveló signos vitales dentro de límites normales. Como hallazgo positivo en el examen abdominal se palpó una masa localizada en el flanco izquierdo, de consistencia firme, bordes bien definidos y de aproximadamente $5 \mathrm{~cm}$ de diámetro, que produjo dolor al tacto. Edema grado I en miembros inferiores. Una ecografía abdominal mostró una masa abdominal gigante de aspecto sólido con áreas hipoecogénicas en probable relación con degeneración quística, la cual parecía respetar bazo y riñón izquierdo. Una tomografía axial computarizada abdominopélvica, sin contraste, mostró una masa tumoral sólida, de densidad heterogénea, localizada en el compartimento retroperitoneal del flanco izquierdo, con dos lobulaciones mediales y diámetros mayores de $7 \times 9 \times 8 \mathrm{~cm}$, independiente de la luz intestinal; mínimo grado de compresión posterior sobre las estructuras del riñón izquierdo sin producir deformidad de los sistemas pielocaliciales ni uréter (figura 1). Los exámenes de laboratorio reportaron hemograma, glicemia y pruebas de función tiroidea dentro de límites normales. Se realizó una laparotomía exploratoria en donde se observó masa bien circunscrita en compartimiento retroperitoneal, realizándose enucleación digital de la misma.

El estudio macroscópico mostró una masa de tejido de $980 \mathrm{~g}$, cuyas medidas fueron 15,5 x 10,5 x $7 \mathrm{~cm}$, de color pardo claro y consistencia firme. Al corte, la superficie era sólida con áreas hemorrágicas y otras cavitadas con contenido gelatinoso de color pardo (figura 2). El examen microscópico mostró múltiples folículos con estructura folicular tiroidea típica, con contenido coloide y algunos con dilatación quística, tapizados por una capa de tirocitos sin atipia y características nucleares regulares (figura 3). Teniendo en cuenta el hallazgo de tejido tiroideo de aspecto usual y el antecedente de ooforectomía por tumor ovárico durante la infancia se hizo el diagnóstico de estrumosis peritoneal. 
Figura 1. TAC simple que muestra una masa tumoral sólida retroperitoneal, con dos lobulaciones mediales y diámetros mayores de 7 × 9 × $8 \mathrm{~cm}$ independiente de la luz intestinal.
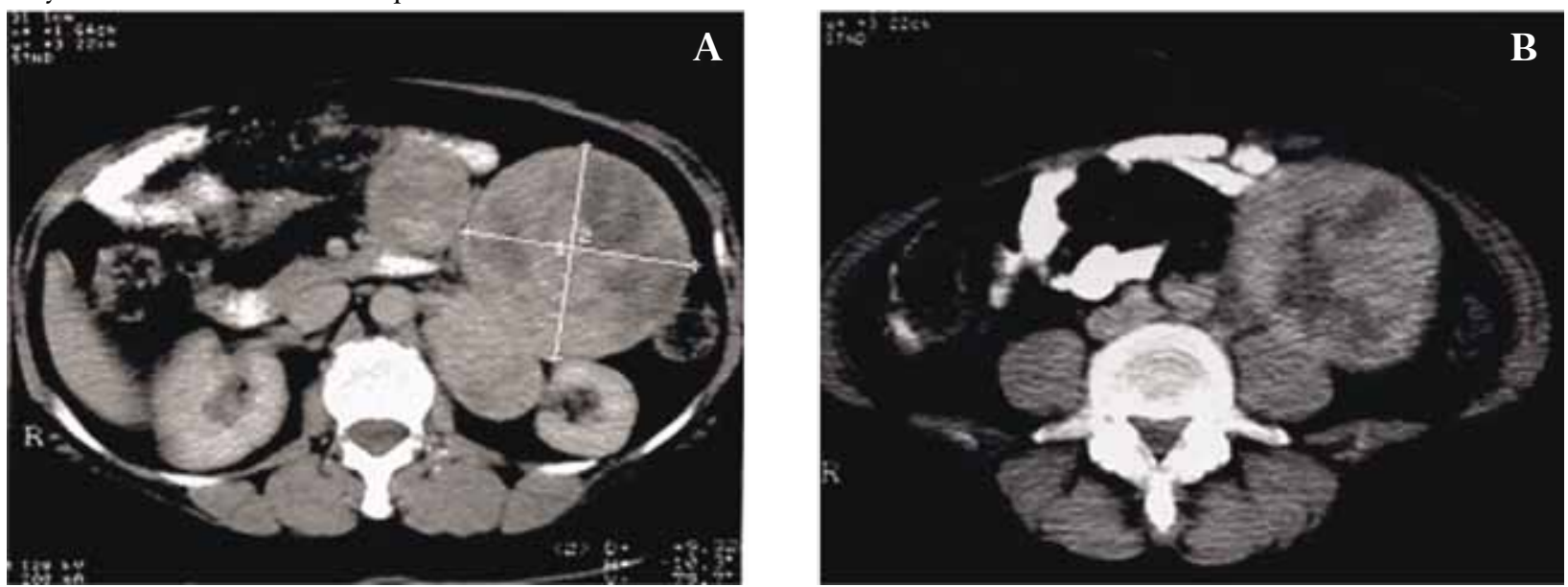

Figura 2. Masa de 15,5 x 10,5 x 7 cm de superficie sólida con áreas hemorrágicas y otras cavitadas con contenido gelatinoso de color pardo.
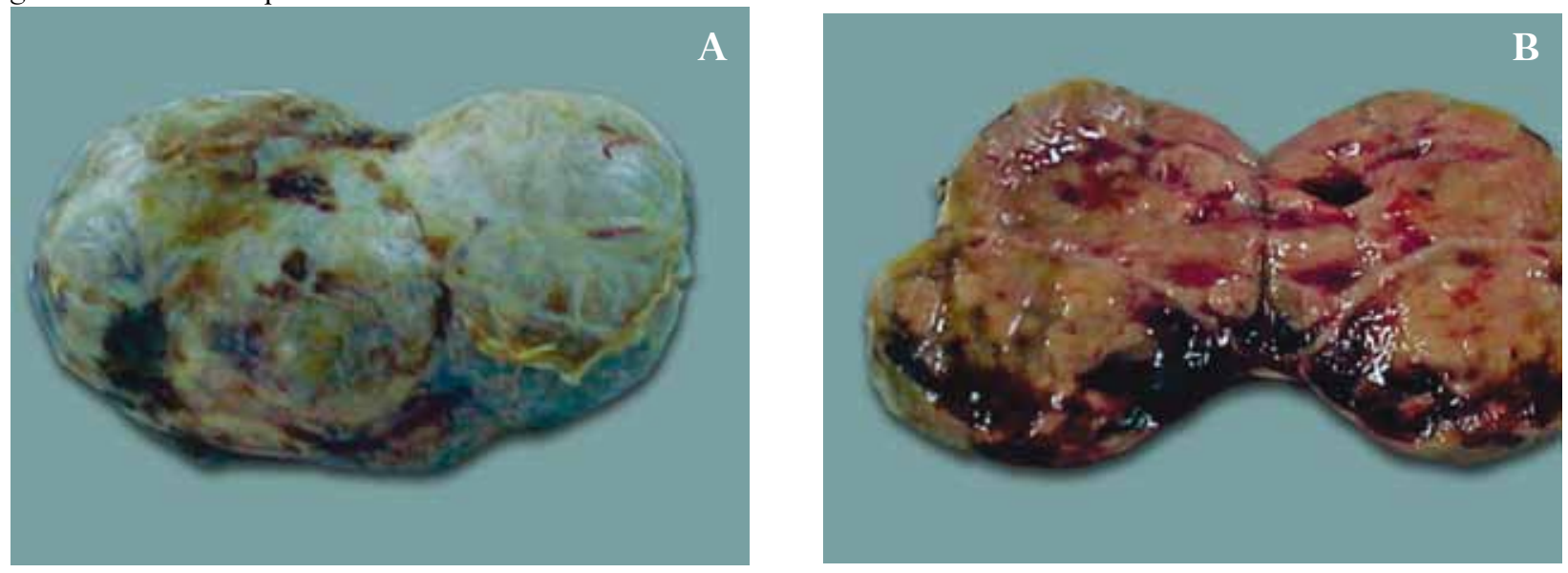

Figura 3. Examen microscópico que reveló múltiples folículos con estructura folicular tiroidea típica, con contenido coloide y algunos con dilatación quística, tapizados por una capa de tirocitos sin atipia y características nucleares regulares.

(H\&E, a. 10X, b. 40X)
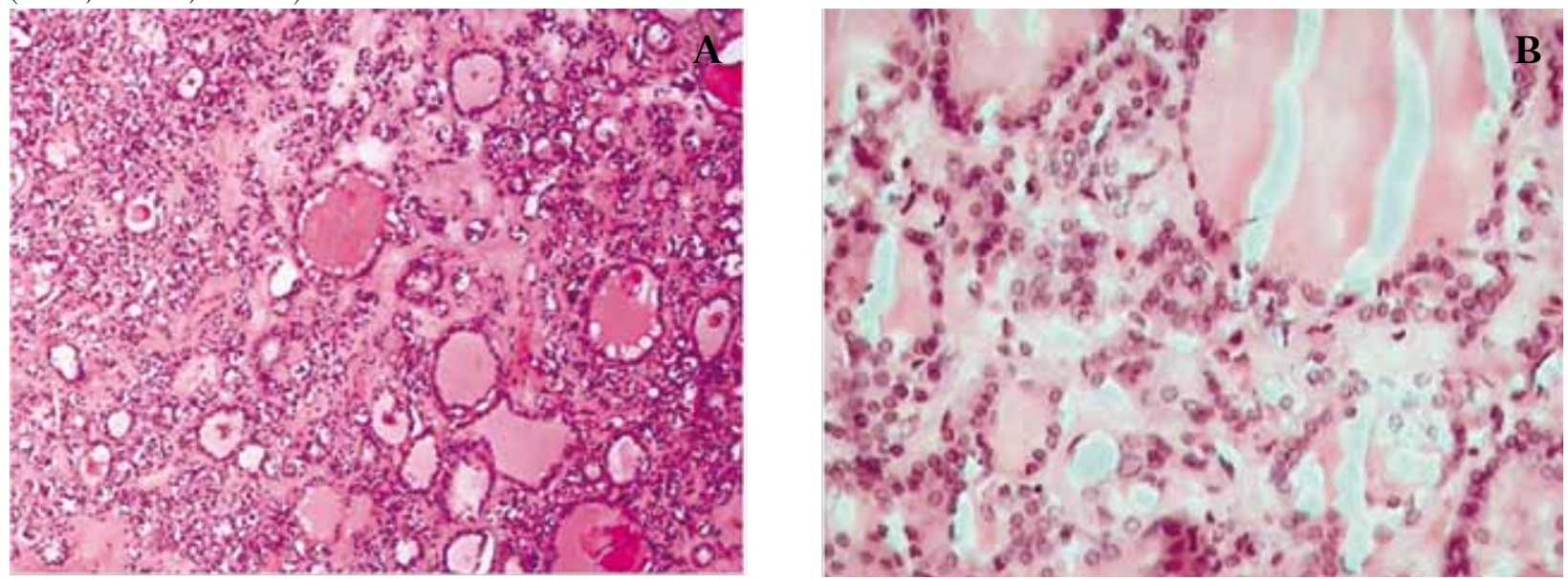


\section{MATERIALES Y MÉTODOS}

Se realizó una búsqueda bibliográfica en las bases de datos Medline vía PubMed, Ebsco, PubMed, Science Direct y Cochrane Reviews, utilizando como encabezados de búsqueda [Título/Resumen]: peritoneal struma, peritoneal strumosis, struma ovarii.

\section{RESULTADOS}

Se encontraron 400 artículos publicados en los últimos 50 años, seleccionando solamente publicaciones en inglés o español que trataran sobre la etiología y diagnóstico de la enfermedad, revisándose un total de 15 artículos.

\section{DISCUSIÓN}

La estrumosis peritoneal presenta asociación patogénica con el estruma ovárico, una variante monodérmica de teratoma ovárico, inicialmente descrita por Bottlin en el año 1888 y luego por Pick en 1902, quienes reconocieron que estaba constituido por tejido tiroideo de aspecto usual; ;,5 este es el teratoma monodérmico ovárico más común y, por definición, debe estar compuesto predominantemente (más del 50\%) por tejido tiroideo. ${ }^{6}$

De forma infrecuente podemos encontrar casos de estruma ovárico con diseminación extraovárica y una apariencia histológica benigna, formando implantes peritoneales benignos en ausencia de un carcinoma somático de tipo tiroideo identificable, es en estos casos en los que se sugieren los términos "estrumosis o estrumatosis" peritoneal., 2,7

La edad de incidencia del estruma ovárico y de los casos de estrumosis peritoneal es la misma que la del teratoma quístico maduro; puede presentarse entre la quinta y sexta década de vida, aunque se conocen casos en mujeres posmenopáusicas y niñas prepúberes. ${ }^{5}$ En este grupo etario los teratomas quísticos maduros son de presentación común ${ }^{4}$ y pueden en raras ocasiones sufrir desgarros capsulares o ruptura del tumor, lo que causa la formación de implantes de tejido tiroideo que se encontraba contenido en el teratoma. ${ }^{3,8}$ Esta es la explicación más aceptada sobre la formación de la estrumosis peritoneal y que concuerda con la historia del caso presentado en esta revisión, en el cual, después de la resección del ovario derecho, la paciente desarrolla la estrumosis de ese mismo lado, sin historia ni hallazgos al examen físico sugestivos de una neoplasia tiroidea. Sin embargo, se han sugerido otras hipótesis que proponen que este fenómeno representa una forma extremadamente bien diferenciada de carcinoma folicular. ${ }^{3}$

Es necesario destacar que aunque el sitio de diseminación más común de un estruma ovárico es el peritoneo, también es posible encontrar implantes en el epiplón, vejiga urinaria y útero. Los implantes generalmente presentan las mismas características macroscópicas que el tumor ovárico original, consistiendo en masas de color pardo con áreas gelatinosas de color verdoso y microscópicamente presentan apariencia de tejido tiroideo con características usuales. ${ }^{9}$ El compromiso de órganos más distales como hígado, pulmones, cerebro y hueso indica malignidad. ${ }^{10}$ Por otra parte, dada la anatomía de los órganos pélvicos y en particular de los ovarios, es posible que solo exista compromiso retroperitoneal, como el presentado en este caso; en esta situación, a pesar de tener el mismo mecanismo patogénico, no existe consenso sobre la utilización del término "estrumosis peritoneal" o "estruma con compromiso extraovárico".

El principal desafío diagnóstico en los casos de estrumosis peritoneal consiste en distinguir esta condición de un carcinoma folicular bien diferenciado con origen en un estruma ovárico y diseminación al peritoneo, este es el segundo tumor maligno más frecuente que se origina de una estrumosis peritoneal, después del carcinoma de tipo papilar. En estos casos es fundamental tener presente que el carcinoma suele comprometer tanto el peritoneo, como los ganglios linfáticos paraaórticos y, en casos más avanzados, el hígado y los pulmones. ${ }^{2,11-13}$ En casos de difícil diferenciación, en donde predomina la apariencia de bocio coloide, no podemos asegurar a simple vista que se trata 
de un proceso benigno y lo que anteriormente era denominado "bocio metastásico benigno", ahora debe llamarse "carcinoma folicular de tipo tiroideo con desviación mínima". ${ }^{5}$ Debido a esto y con el fin de garantizar el bienestar al paciente, se recomienda que el diagnóstico de estrumosis peritoneal se asegure al no encontrar evidencia de progresión neoplásica después de un seguimiento de al menos 5 años. ${ }^{5}$

Las manifestaciones clínicas de este tumor están representadas casi en su totalidad por episodios de dolor abdominal, caracterizándose por un curso clínico indolente; excepcionalmente se encuentran manifestaciones que indiquen funcionalidad del tejido tiroideo, como hipertiroidismo, ${ }^{2,7}$ característica que solo ha sido reportada en estrumosis ovárica por Matsuda y colaboradores. ${ }^{14}$ El manejo de la estrumosis peritoneal depende de la extensión de la enfermedad y de las características histopatológicas de la lesión. El abordaje quirúrgico es usualmente recomendado para lesiones abdominales bien circunscritas. Sin embargo, es necesario tener criterios histopatológicos precisos e identificar cambios proliferativos al evaluar estos tumores, debido a la similitud con carcinoma. Teniendo en cuenta esto, en algunos casos reportados se decidió tratar a estos pacientes con protocolos de cáncer de tiroides, utilizando por lo general radioterapia. $^{8}$

\section{CONCLUSIONES}

Aunque han pasado muchas décadas luego de la primera descripción de un estruma ovárico y algunos casos de estrumosis peritoneal, muchos aspectos aún siguen siendo enigmáticos y el amplio rango de características macroscópicas e histológicas de estos tumores hacen que el correcto diagnóstico y abordaje de estos pacientes sea problemático en ocasiones. Sin importar que nombre se le den a los implantes peritoneales de tejido tiroideo, es necesario estudiar un método reproducible para diferenciar entre una estrumosis peritoneal o un carcinoma folicular de desviación mínima, con el fin de asegurar que se trata de entidades distintas y garantizar el bienestar del paciente.

\section{REFERENCIAS}

1. Karseladze AI, Kulinitch SI. Peritoneal strumosis. Pathol Res Pract 1994;190:1082-5.

2. Hatami M, Breining D, Owers RL, Del Priore G, Goldberg GL. Malignant struma ovarii-a case report and review of the literature. Gynecol Obstet Invest 2008;65:104-7.

3. Costa MA, Póvoa AM, Pires MC, Paiva VL, Pinto C, Martínez-de-Oliveira J. Struma ovarii: a rare form of presentation and clinical review. Acta Obstet Gynecol Scand 2005;84:819-20.

4. Crum CP. Female genital tract-ovarian tumors. En: Cotran RS, Kumar V, Collins T, editors. Robbins Pathologic Basis of Disease. 7th. ed. Philadelphia: Saunders; 2009. p. 963-64.

5. Roth LM, Talerman A. The enigma of struma ovarii. Pathology 2007;39:139-46.

6. Halpenny DF, O’Brien J, Ibrahim MM, Crotty R, Torreggiani WC. An unusual cause of pelvic pain: struma ovarii. JBR-BTR 2009;92:239-41.

7. Tennvall J, Ljungberg O, Högberg T. Malignant struma ovarii' with peritoneal dissemination. Histopathology 1997;31:289-90.

8. Brogsitter C, Wonsak A, Würl K, Kotzerke J. Peritoneal strumosis. Eur J Nucl Med Mol Imaging. 2004;31:1057.

9. Zhang X, Axiotis C. Thyroid-type carcinoma of struma ovarii. Arch Pathol Lab Med 2010;134:786-91.

10. Thomas RD, Batty VB. Metastatic malignant struma ovarii. Two case reports. Clin Nucl Med 1992;17:577-8.

11. Rosai J, Carcangiu ML, DeLellis RA. Tumors of the Thyroid Gland. Washington: Armed Forces Institute of Pathology; 1994.

12. Ruel IF, Fierrard H, Vercellino L, Bernard L, Hindie E, Duron F, et al. Pulmonary metastasis of struma ovarii: a case report. Clin Nucl Med 2010;35:692-4.

13. Osmanağaoğlu M, Bozkaya H, Reis A. Malignant struma ovarii: a case report and review of the literature. Indian J Med Sci 2004;58:206-10.

14. Matsuda K, Maehama T, Kanazawa K. Malignant struma ovarii with thyrotoxicosis. Gynecol Oncol 2001;82:575-7. 